\title{
A Brief Clinical Assessment of Cognitive Deficit with Impaired Daily Living Functioning in Parkinson's Patients with and without Dementia
}

\author{
Sadaf Naeem ${ }^{1}$, Rahila Najam ${ }^{1}$, Nausheen Alam ${ }^{2 *}$, Syed Waseem Akhter ${ }^{3}$ \\ ${ }^{1}$ Department of Pharmacology, Faculty of Pharmacy, University of Karachi, Karachi, Pakistan \\ ${ }^{2}$ Department of Pharmacology, Faculty of Pharmacy, Federal Urdu University, Karachi, Pakistan \\ ${ }^{3}$ Department of Neurology, Karachi Medical and Dental College, Karachi, Pakistan \\ Email: *nausheenasarosh@hotmail.com
}

Received 4 March 2016; accepted 7 May 2016; published 10 May 2016

Copyright (C) 2016 by authors and Scientific Research Publishing Inc.

This work is licensed under the Creative Commons Attribution International License (CC BY). http://creativecommons.org/licenses/by/4.0/

(c) (1) Open Access

\section{Abstract}

Cognitive decline in Parkinson's disease (PD) is defined as dementia which affects activities of daily living (ADL) function. Dementia is one of the recognized issues in the treatment of Parkinson's disease patients (PDP) as it is becoming a major contributor to morbidity. The objective of our study was to evaluate the cognitive decline in Parkinson's disease patients (PDP) and the influence of cognition on daily living function deterioration in cognitively impaired and cognitively unimpaired PDP. Cognitively impaired PDP $(n=24)$, and cognitively unimpaired PDP $(n=16)$ were administered MoCA and MMSE performance based tests for cognitive measurements and Alzheimer's Disease Cooperative Study Activities of Daily Living Inventory (ADCS-ADL) to determine everyday functioning. The significances of MoCA and MMSE among cognitively impaired PDP and cognitively unimpaired $P D P$ in univariant analysis were $P<0.001$ and $P<0.05$. In partial correlation, MoCA was strongly related to ADL function as compare to MMSE $(r=0.623, P=0.001)$. Cognitively impaired PDP showed significantly lower score on visuo-construction and attention. Among all variables of MoCA domains, attention is strongly associated with instrumental activity daily living (IADL) scores (logistic regression coefficient $=0.672, P=0.01$ ). These results suggest that dementia affects daily living performances especially the IADL tasks like attention and execution. Therefore, the assessment of complex daily activities particularly IADL is probably useful for the diagnosis of early stage cognitive impairment in PDP. Further MMSE test is less sensitive than MoCA for detecting significant cognitive decline in PDP and impaired attention is an important determinant of ADL functions in cognitively impaired PDP.

\footnotetext{
${ }^{*}$ Corresponding author.
}

How to cite this paper: Naeem, S., Najam, R., Alam, N. and Akhter, S.W. (2016) A Brief Clinical Assessment of Cognitive Deficit with Impaired Daily Living Functioning in Parkinson's Patients with and without Dementia. Advances in Parkinson's Disease, 5, 15-23. http://dx.doi.org/10.4236/apd.2016.52003 


\section{Keywords}

\section{Parkinson's Disease Patients, Activities of Daily Living, Montreal Cognitive Assessment, Mini Mental State Ex Amination, Instrumental Activity of Daily Living}

\section{Introduction}

Parkinson's disease is a complex type of neurodegenerative disease caused when dopaminergic neurons and nerve terminals that control body movement loss their activity and die [1] [2]. The distinct clinical signs of disease are tremors, postural instability with shuffling gate, bradykinesia and rigidity [3] [4]. Motor deterioration in PD patients is common, and some patients have cognitive decline at a time of diagnosis, average prevalence $80 \%$ which is associated with motor impairment [2] [5]. Gradual motor deterioration produces dementia which highly affects on quality of life and produces difficulty to achieve different tasks of daily living (ADL) in PD patients [6] [7].

The daily living faction of PD patients connected with cognitive impairment, and this relation has not been comprehensively evaluated and may often be missed in clinical practice [8]. Whenever PD is suspected, the routine approach should include a set of questions aimed to explore dementia, and on the other hand, it is useful to quantify their severity and impact [9] [10]. ADL impairment related with deteriorated life feature for patients and for care giver too [11] [12]. The cognitive impairments are a better predictor of every day functional capacity and future disabilities among PD patients than their declining motor abilities [13]. However, activities of daily living are of different types like bathing, eating and getting dressed termed as basic activities of daily life (BADL) which may not change at early dementia in contrast, Instrumental ADL such as using household appliances, reading, conversation or organizing work is more deteriorated even at early stage of dementia [14] [15]. Recent studies showed impaired attention and executive function affect complex activities in PD patients especially the IADL which is related to intact memory [16] [17].

Recent researches have demonstrated the impairment in IADL measures is associated with cognitive status in PD [18]. Some studies have addressed associations between memory or executive functions and instrumental ADL [19] [20] and some explain link between attention deficits in PD patients [21] [22]. Marshal et al. 2010 [23] proposed that detection of ADL changes in Alzheimer's and PD patients were needed to better confine the changes in cognition and behavior. So this is necessary to evaluate complex ADL difficulties especially IADL for better diagnosis of PD associated mild cognitive decline before starting to develop the major dementia. However, no much work has been carried out on the relation between impaired cognitive MoCA domains and difficulties in everyday performances especially the IADL. We hypothesize that PD patients with major cognitive deficit will exhibit greater difficulties in daily life functioning and to achieve tasks than PD patients with no or mild cognitive decline, and second, the MoCA test would be more accurate in diagnosing dementia in PD patients even at early stage of cognitive impairment than MMSE.

\section{Methodology}

Our recruitment period was 16 month during that period we recruited 55 patients who were screened in outpatient department of Neurology of Abbasi Shaheed Hospital Karachi Pakistan. 40 of these patients agreed to participate while 7 patients refused to participate and not given written informed consent and eight others did not meet our study criteria. All recruited patients age were between $45-80$ years, they all were speak and write Ur$\mathrm{du}$ fluently and they were taking L-dopa only to avoid any neuropsychiatric effect of other anti-Parkinson's drug. The United Kingdom Parkinson's Disease Society Brain Bank criteria was used to diagnose Parkinson's disease. Exclusion criteria included the presence of any neurodegenerative disease other than Parkinson's disease or other reason of cognitive decline, medication interfering with cognition (i.e. Hypnotics or tranquilizers), deep brain stimulation, the presence of any disease or debility distinct to Parkinson's disease and the use of a cholinesterase inhibitor or Para sympatholytic drugs during the one month before inclusion in the research.

The study was approved by the Karachi medical dental college ethical review board, Reference \#CHS. 097/11, all PD patients or their informants filled informed consent form before study started. All procedures were conducted in accordance with the ethical standards of the responsible committee on human experimenta- 
tion and with the declaration of Helsinki 2004.

\subsection{Classification of Cognition and ADL Impairment}

The cognitive decline was diagnosed by the neurologist for each PD patient by applying the Diagnostic and Statistical manual mental disorder IV (code 294.1) further MMSE [24] and MoCA [25] were administered by trained staff. The MMSE is a common cognitive test used to assess orientation, verbal memory, language, attention/calculation, and visuo-constructive abilities. however some studies have challenged MMSE efficacy as a screening instrument in Parkinson's disease, because it lacks specific tests for executive function assessment [26] [27], that's why MoCA test also being used to find more accurately cognitive decline in Parkinson's patients. To identify mild to moderate cognitive decline in PD patients, we first identified all patients who had a MMSE score $<26$ and MoCA score $<26$ and declared them cognitively impaired Parkinson's patients and Parkinson's patients who had MMSE \& MoCA score $>26$ considered as cognitively unimpaired.

After determine cognitive status of each patient then at the separate visit to evaluate the daily living performances in PDP "the Alzheimer's Disease Cooperative Study Activities of Daily Living Inventory (ADCS-ADL) scale" was administered by independent rater [28]. For this purpose interview were conducted to patient's informant, in our study most of the informants were their spouse, son or any relative who lives in their home. We asked the informant of the patient filled out the ADCS-ADL scale according to the patient's observed action and behavior, for which scores can range from 0 - 78 points, with lower scores indicating poor performance. ADL scale is contain 23 items, six items like bathing, eating, grooming, walking and getting dressed termed as basic activity of daily living (BADL) maximum score $=22$, and other 17 complex activity items like managing his/her belongings, finances and organizing works etc termed as instrumental activity of daily living(IADL) maximum score $=56[16]$.

\subsection{Motor Examination}

Assessment of Parkinson's patients were carried out by means of motor examination section (part 111) of the Unified Parkinson's Disease Rating Scale (UPDRS) maximum score $=108$ higher score means worst motor symptoms [29] and Hoehn and Yahr staging [30]. These examinations were carried out by movement disorder specialists.

\subsection{Data Analysis}

The data were analyzed by using SPSS version 16.0 for Windows. Comparisons of demographic and clinical characteristics including MoCA and MMSE scores, between cognitively impaired and unimpaired samples were performed using Independent sample $t$-test and univariant analysis. We compared the MoCA domains between both impaired and unimpaired group by using univariant analysis, and correlation between disease severity, duration of disease and cognition, Partial correlation coefficient was used. To predict the affect of cognitive changes and motor fluctuations on ADL function and the comparison of ADL factors (IADL \& BADL) with MMSE and MoCA Partial correlation coefficient was used. Total scores of BADL and IADL between groups were performed using Independent sample $t$-test. Further we evaluated which cognitive subtype is predictor of IADL impairment in PD on the basis of MoCA domains; we applied binary logistic regression analysis. All analysis were conducted at $\mathrm{P}<0.05$ significance level.

\section{Results}

\subsection{Patient's Characteristics}

A total of 40 patients completed all study assessment. The baseline demographical characteristics of the sample population are listed in Table 1.

Table 1 showed no statistically significant differences among both cognitively impaired and unimpaired groups by means of sex, weight, disease duration, age, total daily levodopa dosage, Hoehn and Yahr staging and UPDRS III scores. We found significant less scores in cognitively impaired patients on MMSE and MoCA scale as compare to unimpaired patients, mean (SD) MMSE score 20.08 $\pm 1-81$ in cognitively impaired patients and mean (SD) MMSE score in cognitively unimpaired patients are $27.56 \pm 1-59(95 \% \mathrm{CI},-8.6-6.3) . \mathrm{t}(38) \mathrm{F}=$ $-13.39, \mathrm{P}<0.001$. 


\subsection{Group Differences on Cognitive Domains of MoCA}

Significant differences were found between group comparison on MoCA domains specifically visuo-spatial, execution, language, orientation and delayed recall. On 6 - 7 domains of MoCA Cognitively impaired PD patients showed significant fewer score than unimpaired patients.

Performances of both groups are shown in Table 2.

\subsection{Correlates of Cognitive Impairment}

The Pearson's correlation between older age, duration of disease and sternness of PD with MMSE and MoCA showed strong correlation $\mathrm{P}<0.05$, see Table 3. Age, Hoehn and Yahr stage, duration of disease and UPDRS III were taking as independent variables in Partial correlation coefficient model and MMSE \& MoCA as dependent variables than our results showed strong relationship among these variables with cognitive measures in cognitively impaired PD patients. The results show similar significant correlation of MMSE and MoCA with all above variables.

\subsection{Correlation among Total ADL, Cognitive and Motor Scores}

Table 4 shows Pearson's correlation coefficient among the cognitive measures, total ADL and motor scores. Where ADL was dependent variable and age, duration of disease, MMSE, MoCA UPDRS and Hoehn \& Yahr staging were independent variables. Most of the correlations with cognitively impaired patients were strong, however cognitively unimpaired patients also showed moderate correlation with age, duration of disease and UPDRS scores. That shows cognitively unimpaired PDP also have impact on ADL function. As a cognitive predictor MoCA approached strong correlation with ADL than MMSE (MMSE, $\mathrm{r}=0.581, \mathrm{P}<0.05$ and MoCA $\mathrm{r}=$ $0.623, \mathrm{P}=0.001)$.

\subsection{Cognition and ADL Function Correlations}

Table 5 showed significant correlation between both cognitively impaired and unimpaired group's MMSE \& MoCA scores with daily living scale factors (BADL \& IADL). The MMES \& MoCA show similar partial correlation coefficients in cognitively impaired patients with IADL factors, however the basic ADL impairment did not observe due to cognitive changes in both groups which shows cognitive decline does not affects the basic function of everyday life. After taking MMSE \& MoCA as dependent variables there was a strong association seen between cognitively impaired patient's MoCA and IADL scores for the entire group (partial correlation coefficient $\mathrm{r}=1.00, \mathrm{P}<0.001$, MMSE $\mathrm{r}=0.68, \mathrm{P}<0.05$ ) while basic ADL is also moderately affected (MoCA, $\mathrm{r}=0.612, \mathrm{P}<0.05$, MMSE, $\mathrm{r}=0.411, \mathrm{P}=0.104)$ results demonstrate MoCA showed a significant association with BADL also rather than MMSE in the entire group.

Table 1. Demographic, clinical and neuropsychological characteristics of the two PD groups (cognitively impaired and unimpaired group) $(\mathrm{n}=40)$.

\begin{tabular}{ccccc}
\hline Clinical characteristics & Cognitively impaired group $(\mathbf{n = 2 4 )}$ & Cognitively unimpaired group $(\mathbf{n}=\mathbf{1 6})$ & F-value & P-value \\
\hline Age & $63.79 \pm 8.05$ & $62.19 \pm 9.51$ & 0.404 & $>0.05$ \\
Sex, \% male & $83 \%$ & $76 \%$ & - & - \\
Weight & $71.08 \pm 6.05$ & $69.50 \pm 6.39$ & 0.01 & $>0.05$ \\
Levadopa dose mg/d & $531.67 \pm 204.30$ & $437.50 \pm 230.03$ & 0.35 & $>0.05$ \\
duration of disease & $4.10 \pm 1.37$ & $2.71 \pm 0.99$ & 0.97 & $<0.01$ \\
UPDRSIII score & $32 \pm 2.78$ & $21.31 \pm 2.70$ & 0.16 & $<0.05$ \\
Hoehn \& Yahr staging & $2.06 \pm 0.71$ & $1.84 \pm 0.72$ & 0.1 & $>0.05$ \\
MMSE score & $20.08 \pm 1.81$ & $27.56 \pm 1.59$ & 0.43 & $<0.001$ \\
MoCA score & $16.79 \pm 1.79$ & $25.19 \pm 1.22$ & 2.6 & $<0.001$ \\
ADL score & $45.04 \pm 6.10$ & $50.13 \pm 7.70$ & 1.08 & $<0.05$ \\
\hline
\end{tabular}

Values are mean \pm S.D. Significant differences by student $t$-test. MMSE: Mini mental state examination, MoCA: Montreal cognitive assessment test, ADL: Activities of daily living. 
Table 2. Performance of PD patients on Montreal cognitive assessment (MoCA) domains based on cognitive status.

\begin{tabular}{ccccc}
\hline MoCA Domains & Cognitively impaired group $(\mathbf{n}=\mathbf{2 4})$ & Cognitively unimpaired group $(\mathbf{n}=\mathbf{1 6})$ & F-value & P-value \\
\hline Visuosapatial & $2.52 \pm 1.811$ & $4.44 \pm 0.512$ & 121.772 & $<0.001$ \\
Naming & $2.35 \pm 0.834$ & $2.94 \pm 0.250$ & 19.544 & $<0.001$ \\
Attention & $3.58 \pm 1.41$ & $5.12 \pm 0.342$ & 177.595 & $<0.001$ \\
Language & $1.75 \pm 0.439$ & $2.2 \pm 0.72$ & 10.857 & $<0.001$ \\
Abstraction & $1.40 \pm 0.496$ & $2.4 \pm 0.851$ & 19.21 & $<0.001$ \\
Delayed recall & $3.80 \pm 1.043$ & $5.60 \pm 0.62$ & 364.8 & $<0.001$ \\
Orientation & $4.45 \pm 1.377$ & $5.57 \pm 0.447$ & 59.394 & $<0.001$ \\
\hline
\end{tabular}

Values are mean \pm S.D. Significant differences by student $t$-test.

Table 3. Correlation between age, duration of disease and disease severity with MoCA \& MMSE among cognitively impaired and unimpaired group.

\begin{tabular}{ccccc}
\hline PARAMETERS & Cognitively impaired group $(\mathbf{n}=\mathbf{2 4})$ & \multicolumn{2}{c}{ Cognitively unimpaired group $(\mathbf{n}=\mathbf{1 6})$} \\
\hline Independent variables & MMSE & MoCA & MMSE & MoCA \\
\hline Age & $0.602^{*}$ & $0.739^{* *}$ & $0.586^{*}$ & $0.512^{*}$ \\
Duration of disease & $0.719^{* *}$ & $-0.700^{* *}$ & -0.26 & -0.230 \\
UPDRS & $-0.789^{* *}$ & $-0.820^{* *}$ & 0.282 & -0.370 \\
Hoehn \& Yahr staging & $-0.633^{*}$ & $-0.48^{*}$ & 0.181 & 0.327 \\
\hline
\end{tabular}

Values are Pearson's correlation coefficient $(\mathrm{r}){ }^{*} \mathrm{P}<0.05,{ }^{* *} \mathrm{P}<0.01$.

Table 4. DL measures, partial correlation coefficients for disease severity and cognition.

\begin{tabular}{ccc}
\hline Independent variables ADL scores of cognitively impaired patients $(\mathbf{n}=\mathbf{2 4})$ & ADL scores of cognitively unimpaired patients $(\mathbf{n}=\mathbf{1 6})$ \\
\hline Age & $0.562^{*}$ & $0.528^{*}$ \\
Duration of disease & $-0.714^{* *}$ & $0.57^{*}$ \\
UPDRS & $-0.834^{* *}$ & $0.55^{*}$ \\
Hoehn \& Yahr staging & 0.118 & 0.297 \\
MMSE & $0.581^{*}$ & -0.396 \\
MoCA & $0.623^{*}$ & -0.255 \\
\hline
\end{tabular}

Values are Pearson's correlation coefficient $(\mathrm{r}){ }^{*} \mathrm{P}<0.05,{ }^{* *} \mathrm{P}<0.01$.

Table 5. Partial correlation coefficients for the association between cognitive measures (MMSE \& MoCA) and ADL factors.

\begin{tabular}{ccccc}
\hline & Cognitively impaired group $(\mathbf{n}=\mathbf{2 4})$ & \multicolumn{2}{c}{ Cognitively unimpaired group $(\mathbf{n}=\mathbf{1 6})$} \\
\hline Cognitive tests & BADL & IADL & BADL & IADL \\
\hline MoCA & $0.612^{*}$ & $1.00^{* *}$ & 0.337 & 0.326 \\
MMSE & 0.411 & $0.681^{*}$ & 0.051 & 0.001 \\
\hline
\end{tabular}

Values are Pearson's correlation coefficient (r) ${ }^{*} \mathrm{P}<0.05,{ }^{* *} \mathrm{P}<0.01$.

\subsection{Results of the Logistic Regression Analysis}

Figure 1 shows the bar graph of total BADL \& IADL scores in cognitively impaired and unimpaired Parkinson's disease patients

Binary Logistic regression analysis was conducted to explore MoCA total score and their domains association to BADL \& IADL. where BADL \& IADL were dependent variables and MoCA domains were independent variables, Most of the independent variables like attention and execution did not show significant association with BADL except delayed recall and orientation (regression coefficient $=0.618, \mathrm{P}=0.01$ ) On the other hand, among all variables attention is strongly associated with IADL scores (regression coefficient $=0.672, \mathrm{P}=0.01$ ), Hosmer and Leme show goodness of fit test; chi-square $=1.3$ and $\mathrm{P}=0.01$. This showed that attention along with 


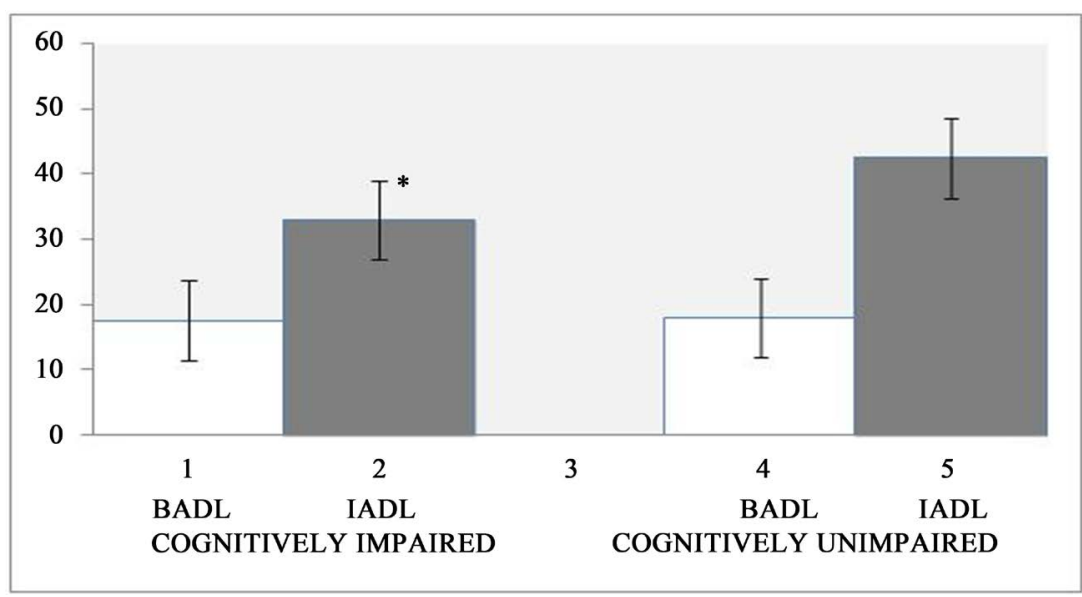

Values are mean \pm S.D. Significant differences by student $t$ - test ${ }^{*} \mathrm{P}<0.05$ BADL (Basic activities of daily living), IADL (Instrumental activities of daily living).

Figure 1. Total basic ADL \& instrumental ADL scores in cognitive impaired and unimpaired Parkinson's patients.

execution had a significant influence on the IADL variation.

\section{Discussion}

Our study confirmed that difficulty in ADL function is strongly correlated to cognitive decline in PDP. This finding is consistent with previous studies which explain the relationship of cognitive decline with ADL dysfunction and proofs everyday life tasks greatly affected due to cognitive impairment in PDP. Moreover in this study we compared the usefulness of the MMSE and MoCA to identify significant cognitive impairment in PDP and relationship of the MoCA and its domains with ADL factors. A 10 minutes dementia detecting test MoCA is very sensitive to detect worst cognitive status in PD patients [31] [27]. To our knowledge very few previous studies identifying this relationship to explore the early stage cognitive impairment and its relationship to ADL in PD patients.

In our clinical study, our small sample size was warranting us to take caution in interpreting the results. In general, estimates based on small numbers of interpretations are less reliable than those based on larger ones. We would have the capacity to identify a moderately large effect size of 0.78 at a significant level of 0.01 . We did discover a significant improve results with MoCA than MMSE test in predicting dementia in PD patients, particularly in the case of slightly impaired subjects. This finding proposes that, at any rate in this little population size, the efficacy of MoCA was clinically significant. This result is consistent with previous observations that the MMSE is not sensitive to predict mild cognitive deficit [32] [27]. Furthermore, according to Trzepacz et al. (2015) [33] MoCA test is slightly more sensitive to detect earlier symptoms of memory loss than MMSE. We found approximately 30\% of PD patients whose MMSE score was normal but they showed significant low MoCA scores which indicate sensitivity of MoCA test as compare to MMSE. This finding suggest MoCA test should prefer in routine clinical practice to detect early stage cognitive status in PDP which clinically undiagnosed and being not detected due to less sensitivity of MMSE test.

In Table 3, partial correlation analysis between ages, male gender, duration of disease and UPDRS with MoCA and MMSE, showed significant low MoCA scores. These results confirmed with previous studies which reported the same variables have also been involved in the development of cognitive impairment in PD patients [34] [31]. This finding propose if patient diagnosed with early stage of dementia by any specific cognitive screening test like MoCA than he will might be a better candidate to start his treatment with pharmacological and non pharmacological means.

Furthermore, we found a link between cognitive impairment, motor abnormalities and ADL performances. Our data shows not even cognitively impaired but cognitively unimpaired PDP also showed association between total ADL, UPDRS motor scores and duration of disease which explain ADL deterioration present not only in cognitively impaired but cognitively unimpaired PDP also have difficulty to perform their daily tasks. Additionally several researchers demonstrates that impairment in instrumental ADL is a stronger predictor of cognitive 
decline [35]-[37]. We explore the same strong relationship between MoCA and IADL function, in Table 5 we can seen cognition and every day functioning both are interconnected and depends on IADL. Previous studies proof our findings which showed complex IADL closely linked with cognitive status especially on attention and gradually fluctuate with cognitive scarcity, contrary basic ADL of PD patients does not affected [16] [38] [39].

Perhaps the most difficult aspect of our study was to analyze the MoCA scores among cognitive impaired and unimpaired PDP and relationship of MoCA domains to ADL factors (BADL \& IADL). By comparing MoCA domains to IADL \& BADL scores we found significant regression coefficient $=0.672, \mathrm{P}=0.001$ with $\mathrm{IADL}$ and showed strong link with Attention and Visio-spatial abilities which reveal complex every day functioning such as organizing work and using household appliances are dependent on attention and visuo-spatial abilities. While previous work demonstrates only attention was the strongest predictor for the severity of ADL impairment [7] [16] [17]. These findings emphasize the importance to diagnose IADL impairment which is a crucial part and indicator of cognitive changes in PD patients and should be checked in regular clinical practice.

In our study we have several limitations. We obtained motor scoring of all PDP while they were taking their regular scheduled dopaminergic doses, motor disability may not be perfectly detected during patient's medication "ON" stage. For this reason we also used Hoehn and Yahr staging to adjust the motor impairment. Further we did not use any inclusive neuropsychological battery to assess cognitive changes in PD patients. We used MoCA for specific cognitive assessment since there were several limitations to the MoCA. This instrument required the participants to follow verbal and written commands; hence the performance of elderly with hearing or visual impairment would be affected. Illiterate or poorly educated persons might have difficulty in comprehending the instructions and the cube and clock drawing tasks were too difficult.

\section{Conclusions}

Our data suggest that before diagnosis of expected cognitive changes in PD patients, the ADL difficulties have been already present and patients have been suffered with daily impaired tasks especially complex IADL tasks. It may be important in clinical practice to rule out early dementia in PD patients by using most sensitive cognitive screening tool like MoCA. According to our results, MoCA was more specific in determining mild cognitive impairment rather than MMSE. Further studies in large cohort with long duration of assessment may give better results in assessing validity of these tests.

Our study suggests daily living performances especially the IADL tasks deteriorate, which is related to attention and execution. Therefore, the assessment of complex daily activities particularly IADL is probably useful for the diagnosis of early stage cognitive impairment in PDP. Other future research studies on larger PD groups will allocate more definite characterization $\&$ recognition of worse cognitive status and its relationship to IADL at early phase of disease will facilitate clinician to inform PD patients and their family about disease prospects and minimize the progression of disease as well as cognitive decline which ultimately improves daily life performances.

\section{Acknowledgements}

We would like to acknowledge Dr. Rahila Najam and Dr. Syed Waseem Akhter for their assistance with recruiting individuals with PD. We would like to thank all of the research participants for their time and willingness to take part in this study. We would also like to thank Dr. Nausheen Alam, Dr. Nadeem and Dr. Nuzhat for assisting with data collection, Finally Dr. Muslehuddin Kalar for assisting with statistical analysis.

\section{References}

[1] Fahn, S. and Przedborski, S. (2000) Parkinsonism. In: Rowland, L.P., Ed., Parkinsonism in Merritt's Neurology, Lippincott, Williams \& Wilkins, New York, 679-693.

[2] Sandhu, K.S. and Rana, A.C. (2015) Evaluation of Anti Parkinson's Activity of Nigella Sativa (Kalonji) Seeds in Chlorpromazine Induced Experimental Animal Model. International Journal of Pharmacy and Pharmaceutical Sciences, $\mathbf{5}, 3$.

[3] Homykiewicz, O. (1998) Biochemical Aspects of Parkinson's Disease. Neurology, 51, S2-S9. http://dx.doi.org/10.1212/WNL.51.2 Suppl 2.S2

[4] Meireles, J. and Massano, J. (2012) Cognitive Impairment and Dementia in Parkinson's Disease: Clinical Features, 
Diagnosis, and Management. Frontiers in Neurology, 3, 88. http://dx.doi.org/10.3389/fneur.2012.00088

[5] Aarsland, D., Litvan, I., Salmon, D., Galasko, D., Wentzel-Larsen, T. and Larsen, J.P. (2003) Performance on the Dementia Rating Scale in Parkinson's Disease with Dementia and Dementia with Lewy Bodies: Comparison with Progressive Supranuclear Palsy and Alzheimer's Disease. Journal of Neurology, Neurosurgery, and Psychiatry, 74, 12151220. http://dx.doi.org/10.1136/jnnp.74.9.1215

[6] Lang, A.E. and Lozano, A.M. (1998) Parkinson's Disease: First of Two Parts. The New England Journal of Medicine, 339, 1044-1053. http://dx.doi.org/10.1056/NEJM199810083391506

[7] Bronnick, K., Ehrt, U. and Emre, M. (2006) Attentional Deficits Affect Activities of Daily Living in Dementia-Associated with Parkinson's Disease. Journal of Neurology, Neurosurgery, and Psychiatry, 77, 1136-1142. http://dx.doi.org/10.1136/jnnp.2006.093146

[8] Muslimovic, D., Post, B., Speelman, J.D., Schmand, B. and de Haan, R.J. (2008) Determinants of Disability and Quality of Life in Mild to Moderate Parkinson Disease. Neurology, 70, 2241-2247. http://dx.doi.org/10.1212/01.wnl.0000313835.33830.80

[9] Schrag, A. and Schott, J.M. (2006) Epidemiological, Clinical, and Genetic Characteristics of Early-Onset Parkinsonism. The Lancet Neurology, 5, 355-363. http://dx.doi.org/10.1016/S1474-4422(06)70411-2

[10] Schrag, A., Jahanshahi, M. and Quinn, N. (2000) What Contributes to Quality of Life in Patients with Parkinson's Disease? Journal of Neurology, Neurosurgery, and Psychiatry, 69, 308-312.

[11] Aarsland, D., Larsen, J.P., Karlsen, K., Lim, N.G. and Tandberg, E. (1999) Mental Symptoms in Parkinson's Disease Are Important Contributors to Caregiver Distress. International Journal of Geriatric Psychiatry, 14, 866-874. http://dx.doi.org/10.1002/(SICI)1099-1166(199910)14:10<866::AID-GPS38>3.0.CO;2-Z

[12] Chaudhuri, K.R. and Schapira, A.H. (2009) Non-Motor Symptoms of Parkinson's Disease: Dopaminergic Pathophysiology and Treatment. The Lancet Neurology, 8, 464-474.

[13] Young, T.L., Granic, A., Chen, T.Y., Haley, C.B. and Edwards, J.D. (2010) Everyday Reasoning Abilities in Persons with Parkinson's Disease. Movement Disorders: Official Journal of the Movement Disorder Society, 25, $2756-2761$. http://dx.doi.org/10.1002/mds.23379

[14] Albert, S.M., Bear-Lehman, J. and Burkhardt, A. (2009) Lifestyle-Adjusted Function: Variation beyond BADL and IADL Competencies. The Gerontologist, 49, 767-777. http://dx.doi.org/10.1093/geront/gnp064

[15] Marshall, G.A., Amariglio, R.E., Sperling, R.A. and Rentz, D.M. (2012) Activities of Daily Living: Where Do They Fit in the Diagnosis of Alzheimer's Disease? Neurodegenerative Disease Management, 2, 483-491. http://dx.doi.org/10.2217/nmt.12.55

[16] Rosenthal, E., Brennan, L., Xie, S., Hurtig, H. and Milber, J. (2010) Association between Cognition and Function in Patients with Parkinson Disease with and without Dementia. Movement Disorders, 25, 1170-1176. http://dx.doi.org/10.1002/mds.23073

[17] Liepelt-Scarfone, I., Fruhmann Berger, M., Prakash, D., Csoti, I., Gräber, S., Maetzler, W. and Berg, D. (2013) Clinical Characteristics with an Impact on ADL Functions of PD Patients with Cognitive Impairment Indicative of Dementia. PLOS ONE, 8, e82902. http://dx.doi.org/10.1371/journal.pone.0082902

[18] Pirogovsky, E., Schiehser, D.M., Obtera, K.M., Burke, M.M., Lessig, S.L., Song, D.D., Litvan, I. and Filoteo, J.V. (2014) Instrumental Activities of Daily Living Are Impaired in Parkinson's Disease Patients with Mild Cognitive Impairment. Neuropsychology, 28, 229-237. http://dx.doi.org/10.1037/neu0000045

[19] Foster, E.R. (2014) Instrumental Activities of Daily Living Performance among People with Parkinson's Disease without Dementia. The American Journal of Occupational Therapy, 68, 353-362. http://dx.doi.org/10.5014/ajot.2014.010330

[20] Cahn-Weiner, D.A., Farias, S.T., Julian, L., Harvey, D.J., Kramer, J.H., Reed, B.R. and Chui, H. (2007) Cognitive and Neuroimaging Predictors of Instrumental Activities of Daily Living. Journal of the International Neuropsychological Society: JINS, 13, 747-757. http://dx.doi.org/10.1017/s1355617707070853

[21] Zhou, W., Hohmann, A.G. and Crystal, J.D. (2012) Rats Answer an Unexpected Question after Incidental Encoding. Current Biology, 22, 1149-1153. http://dx.doi.org/10.1016/j.cub.2012.04.040

[22] Dujardin, K., Leentjens, A.F.G., Langlois, C., Moonen, A.J.H., Duits, A.A., Carette, A.-S. and Duhamel, A. (2013) The Spectrum of Cognitive Disorders in Parkinson's Disease: A Data-Driven Approach. Movement Disorders, 28, $183-$ 189. http://dx.doi.org/10.1002/mds. 25311

[23] Marshall, G.A., Rentz, D.M. and Frey, M.T. (2011) Executive Function and Instrumental Activities of Daily Living in Mild Cognitive Impairment and Alzheimer's Disease. Alzheimer's \& Dementia: The Journal of the Alzheimer's Association, 7, 300-308. http://dx.doi.org/10.1016/j.jalz.2010.04.005

[24] Folstein, M.F., Folstein, S.E. and McHugh, P.R. (1975) Mini-Mental State: A practical Method for Grading the Cogni- 
tive State of Patients for the Clinician. Journal of Psychiatric Research, 12, 189-198. http://dx.doi.org/10.1016/0022-3956(75)90026-6

[25] Nasreddine, Z.S., Phillips, N.A., Bedirian, V., Charbonneau, S., Whitehead, V. and Collin, I. (2005) The Montreal Cognitive Assessment, MoCA: A Brief Screening Tool for Mild Cognitive Impairment. Journal of the American Geriatric Society, 53, 695-699. http://dx.doi.org/10.1111/j.1532-5415.2005.53221.x

[26] Mamikonyan, E., Moberg, P.J., Siderowf, A., Duda, J.E., Have, T.T., Hurtig, H.I., Stern, M.B. and Weintraub, D. (2009) Mild Cognitive Impairment Is Common in Parkinson's Disease Patients with Normal Mini-Mental State Examination (MMSE) Scores. Parkinsonism \& Related Disorders, 15, 226-229. http://dx.doi.org/10.1016/j.parkreldis.2008.05.006

[27] Nazem, S., Siderowf, A.D., Duda, J.E., Have, T.T. and Colcher, A. (2009) Montreal Cognitive Assessment Performance in Patients with Parkinson's Disease with "Normal" Global Cognition According to Mini-Mental State Examination Score. Journal of the American Geriatrics Society, 57, 304-308. http://dx.doi.org/10.1111/j.1532-5415.2008.02096.x

[28] Galasko, D., Bennett, D.A. and Sano, K. (1997) An Inventory to Assess Activities of Daily Living for Clinical Trials in Alzheimer's Disease. Alzheimer Disease \& Associated Disorders, 2, 33-39. http://dx.doi.org/10.1097/00002093-199700112-00005

[29] Fahn, S. and Elton, R.L. (1987) Unified Parkinson's Disease Rating Scale. In: Fahn, S., Marsden, C.D., Calne, D. and Goldstein, M., Eds., Recent Developments in Parkinson's Disease, Macmillan Health Care Information, Florham Park, 153-164.

[30] Hoehn, M.M. and Yahr, M.D. (1967) Parkinsonism: Onset, Progression and Mortality. Neurology, 17, 427-442. http://dx.doi.org/10.1212/WNL.17.5.427

[31] Gill, D.J., Freshman, A., Blender, J.A. and Ravina, B. (2008) The Montreal Cognitive Assessment as a Screening Tool for Cognitive Impairment in Parkinson's Disease. Movement Disorders, 23, 1043-1046.

[32] Faustman, W.O., Moses, J.A. and Csernansky, J.G. (1990) Limitations of the Mini-Mental State Examination in Predicting Neuropsychological Functioning in a Psychiatric Sample. Acta Psychiatrica Scandinavica, 81, 126-131. http://dx.doi.org/10.1111/j.1600-0447.1990.tb06464.x

[33] Trzepacz, P.T., Hochstetle, H., Wang, S., Walker, B. and Saykin, A.J. (2015) Relationship between the Montreal Cognitive Assessment and Mini-Mental State Examination for Assessment of Mild Cognitive Impairment in Older Adults. BMC Geriatrics, 15, 107. http://dx.doi.org/10.1186/s12877-015-0103-3

[34] Emre, M., Aarsland, D., Brown, R., Burn, D.J., Duyckaerts, C., et al. (2007) Clinical Diagnostic Criteria for Dementia Associated with Parkinson's Disease. Movement Disorders, 22, 1689-1707. http://dx.doi.org/10.1002/mds.21507

[35] Kayoko, M. (2016) Neuropsychological Characteristics and Their Association with Higher-Level Functional Capacity in Parkinson's Disease. Dementia and Geriatric Cognitive Disorders Extra, 5, 271-284.

[36] Perneczky, R., Pohl, C., Sorg, C., Hartmann, J., Tosic, N., Grimmer, T., et al. (2006) Impairment of Activities of Daily Living Requiring Memory or Complex Reasoning as Part of the MCI Syndrome. International Journal of Geriatric Psychiatry, 21, 158-162.

[37] Ahn, I.S., Kim, J.H., Kim, S., Chung, J.W., Kim, H., Kang, H.S. and Kim, D.K. (2009) Impairment of Instrumental Activities of Daily Living in Patients with Mild Cognitive Impairment. Psychiatry Investigation, 6, 180-184.

[38] Rasovska, H. and Rektorova, I. (2011) Instrumental Activities of Daily living in Parkinson's Disease Dementia as Compared with Alzheimer's Disease: Relationship to Motor Disability and Cognitive Deficits: A Pilot Study. Journal of the Neurological Sciences, 310, 279-282.

[39] Jekel, K., Damian, M., Wattmo, C., Hausner, L., et al. (2015) Mild Cognitive Impairment and Deficits in Instrumental Activities of Daily Living: A Systematic Review. Alzheimer's Research \& Therapy, 7, 17. 